(Se) former pour enseigner le français à ceux qui ne le parlent pas nativement -

\title{
Rahul Markovits. Civiliser l'Europe. Politiques du théâtre français au XVIIIe siècle.
}

\section{Nadia Minerva}

\section{(2) OpenEdition \\ Journals}

Édition électronique

URL : https://journals.openedition.org/dhfles/4337

DOI : $10.4000 /$ dhfles.4337

ISSN : 2221-4038

Éditeur

Société Internationale pour l'Histoire du Français Langue Étrangère ou Seconde

Édition imprimée

Date de publication : 1 décembre 2015

Pagination : 213-221

ISSN : 0992-7654

Référence électronique

Nadia Minerva, «Rahul Markovits. Civiliser I'Europe. Politiques du théâtre français au XVIIIe siècle. », Documents pour l'histoire du français langue étrangère ou seconde [En ligne], 55 | 2015, mis en ligne le 19 novembre 2017, consulté le 25 mars 2023. URL : http://journals.openedition.org/dhfles/4337 ; DOI : https://doi.org/10.4000/dhfles.4337

Ce document a été généré automatiquement le 25 mars 2023.

Tous droits réservés 


\title{
Rahul Markovits. Civiliser l'Europe. Politiques du théâtre français au XVIIIe siècle.
}

\author{
Nadia Minerva
}

Paris : Fayard, coll. « L'épreuve de l'histoire », 2014, 400 p. EAN : 9782213681375

2 Le verbe " franciser " acquiert aux XVII et ${ }^{e} \mathrm{XVIII}^{\mathrm{e}}$ siècles un sens plus large par rapport à la signification strictement linguistique qu'il avait depuis qu'il était en usage, au XVI ${ }^{\mathrm{e}}$ siècle («franciser un mot»). À Hanovre, réside jusqu'en 1757 un théâtre français entretenu par les électeurs (à partir de 1714 également rois d'Angleterre). Pendant l'occupation française d'Hanovre, ayant duré six mois en 1757-58, on exploite la troupe de «sa Majesté Britannique » pour des représentations, relate, dans le Journal militaire, son rédacteur François-Antoine Chevrier. C'est dans ce contexte qu'apparaît le mot «franciser » pour désigner un processus d'acculturation (p. 191) : le mot est référé à l'action exercée par les Français sur les habitants d'Hanovre, puis, à la faveur d'une invasion hypothétique, sur les Anglais. Ce mot - relève Markovits (note 109, p. 347) est absent de la première édition du Manuel lexique ou Dictionnaire portatif des mots français de l'abbé Prévost (1750) : il figure en revanche dans le supplément de 1755 (avec le sens de "rendre français »); il s'impose donc en Europe dans les années 1750. En appui aux arguments de Markovits, ajoutons que le mot ne figure pas non plus dans le Dictionnaire de l'Académie où il n'est enregistré qu'à partir de la deuxième édition de 1762, dans les deux sens, linguistique et culturel. Pour ce dernier on donne la définition suivante : «Il se dit aussi en parlant Des personnes, \& ne s'emploie qu'avec le pronom personnel, pour dire, que Quelqu'un prend l'air, le maintien, les manières Françoises. Cet Étranger s'est bien francisé depuis trois mois qu'il est à Paris." On le voit, pour le lexicographe, il désigne une action exercée sur un individu séjournant en France et non pas sur des collectivités, que ce soient les Hanovriens ou les Anglais, dont il faut changer les mœurs. En Nouvelle-France - nous rappelle l'A. - ce mot date des années 1660 et est employé par les religieux et les administrateurs pour désigner l'œuvre de civilisation des natifs. "Franciser» signifie donc "civiliser» les Hanovriens et les 
Anglais, comme les sauvages d'Amérique. Quel rôle joue le théâtre français dans cette tâche assumée par les Français de civiliser l'Europe?

Plus de 5000 pièces représentées dans à peine cinq théâtres (de Berlin, Parme, Vienne, La Haye et Stockholm), 81 villes accueillant une troupe française, 25 théâtres permanents, des centaines de troupes sillonnant l'Europe de Cadix à Moscou, un chiffre d'affaires millionnaire, des frais immenses supportés par les États étrangers. Des chiffres à donner le tournis pour documenter la présence du théâtre français à l'étranger au XVIII ${ }^{e}$ siècle. Une présence qui a été interprétée comme le signe de la propagation illimitée de la culture française, mais dont les coordonnées étaient fumeuses. Dès le premier chapitre, Markovits répertorie les lieux réels de ce rayonnement pour dessiner une nouvelle carte de l'«Europe française " destinée à remplacer celle aux contours impressionnistes et mythiques esquissée en 1777 par le comte Caraccioli quand il inventait l'expression dans son Paris le modèle des nations étrangères ou l'Europe française. Précisant la carte, Markovits précise aussi la chronologie : un graphique (p. 31) montre trois phases d'expansion aux alentours de 1710,1740 et 1760 , avant la décrue progressive du nombre de troupes françaises à l'étranger. Si les données recueillies par Markovits confirment les études sur l'Europe française, l'auteur n'est pas dupe de ce «mythe » déroutant : son point de vue renverse celui de Brunot, Réau, Fumaroli et de tous ceux qui se sont mis sur les traces de Caraccioli. Si un sillon se creuse entre lui-même et ses prédécesseurs, affirme-t-il, c'est à cause de la double marque d'ubiquité et d'uniformité (p. 30) dont on a dénoté la présence de la culture française à l'étranger. Dès lors, la tâche de Markovits est de nuancer, distinguer, préciser. En effet, le but déclaré de ce livre est un changement de perspective : ce livre met l'accent « sur la part créatrice de la réception » (p. 21), étudie les transferts culturels et le réseau d'interdépendances dynamiques entre toutes les composantes. L'idée que le théâtre français était un véritable instrument d'acculturation, idée de sa mission civilisatrice que Caraccioli avait héritée de Voltaire, est donc, selon Markovits, une simplification qui cache la complexité de phénomènes que seule l'histoire transnationale peut aider à comprendre.

4 Le livre de Markovits comprend deux parties ; la première décrit l'expansion du théâtre français dans les cours princières au XVIII ${ }^{\mathrm{e}}$ siècle, quand les troupes de comédiens traversent l'Europe en jouant un répertoire français, disséminant à l'étranger ce théâtre qui est vu par les élites françaises comme la quintessence de la civilisation qu'elles prétendent incarner. Cette première partie dessine l'espace européen du théâtre français, décrit le marché du travail qu'il engendre, s'interroge sur ce que devient le répertoire français sur les scènes étrangères et met à nu, enfin, les enjeux du projet politique-culturel qui sous-tend, d'un côté et de l'autre, l'exportation du théâtre et son appropriation/acclimatation à l'étranger. La deuxième partie indique un parcours - visant de plus en plus à imposer la culture française - qui va de la politique de la "puissance douce» du théâtre aux armées à l'« impérialisme culturel» dans l'« exportation de la Révolution" par la conquête, passant par la querelle entre les Philosophes sur Genève et aboutissant à la régénération/acculturation des départements annexés.

5 Le théâtre français est surtout présent dans les cours princières de l'Europe du centre et du nord, et ne touche que marginalement les publics citadins de ces régions, de même que ne sont pas touchées l'Angleterre et l'Espagne, pays dont le théâtre national est glorieux. Il est joué aussi bien par des troupes françaises que par les aristocrates (v. 
le paragraphe «L'aristocratie sur les planches », p. 127-130, consacré aux performances théâtrales des courtisans de tout âge et sexe, à Stockholm et à Vienne notamment) : la fonction éducative du théâtre est prisée à l'époque : elle tient à l'apprentissage de la langue française et de l'expression corporelle. Très souvent, parmi les motivations justifiant le recrutement de comédiens français, est en évidence celle de rendre plus familière la langue française.

6 Véritables citoyens européens, les comédiens évoluent au gré d'un marché en mouvement (leurs profils et leurs déplacements sont restitués par les passeports conservés dans les archives). Leur carrière a une dimension internationale et Markovits étudie leurs migrations, leurs conditions de vie et d'engagement, leurs appointements. Les comédiens sont tenus pour des agents culturels, le théâtre - pratique autant sociale que littéraire préférée des élites - est l'arme civilisatrice et la langue, porteuse d'un art de vivre, est le symbole de cette civilisation. Mais si une troupe d'acteurs venue de France est pour un prince un élément de prestige (la fonction des spectacles étant de "dégager des bénéfices d'ordre symbolique», p. 37), elle est aussi une dépense coûteuse. La gestion du théâtre français à l'étranger est toujours économiquement déficitaire. La presque simultanéité des décisions de congédier les troupes françaises (à Parme, à Stockholm, à la cour polonaise et à Vienne, aux alentours de 1770) montre quel est le poids des motivations politiques dans la gestion des spectacles : la « logique somptuaire » des cours princières européennes entre en crise à partir des années 1760 . Markovits prouve ainsi l'inconsistance de l'idée selon laquelle il faut voir une "spontanéité » inéluctable dans la diffusion de l'influence française, "portée par le double prestige versaillais et parisien, et au-delà par le "génie" universel de la langue française » (p. 91). Les coûts sont une de ces contraintes matérielles auxquelles est sujet le théâtre français à l'étranger, contraintes que l'A. s'efforce de mettre en avant pour inviter à reconsidérer l'opinion courante sur l'Europe française. Une autre idée reçue est l'étendue des compétences en français des élites européennes.

7 «Sourcils froncés et [...] poings serrés ", « habit sarmate en fourrure, brodé d'or et retenu par une ceinture de soie chatoyante » (p. 102), le portrait - en couverture - du comédien Monvel jouant Ladislas dans Venceslas de Rotrou est un peu l'emblème de "l'attrait muet» du spectacle français à l'étranger: si le public ne comprend les dialogues de la pièce que de manière approximative, le charme des costumes somptueux et l'attitude farouche et menaçante du comédien suffisent à retenir l'attention des spectateurs. Cette évocation du portrait d'un des comédiens au service de la cour de Suède dans les années 1780 est au cœur des arguments de Markovits qui s'interroge, au chapitre 3, sur l'accueil des pièces françaises lorsqu'elles passent les frontières. En effet, la première question à laquelle il cherche à répondre est à quel point le public comprend ce qui se passe sur scène. Si on a interprété la présence des comédiens français à l'étranger comme la preuve de la francophonie irréfutable du public, donc d'une Europe française profonde et capillaire, ce livre vise à soutenir que tout au contraire - le décalage linguistique est un facteur bien connu des responsables locaux de la programmation des spectacles. Cronström, chargé de recruter une troupe française pour la cour de Suède, suggère à son correspondant en France de procurer " une trouppe meslée de musiciens et danseurs », doutant des compétences en français du jeune Charles XII et de ses courtisans (p. 103 : Cronström à Tessin, 3 mai 1699). À Vienne, Durazzo, directeur général des spectacles, préconise des représentations avec des ballets pour attirer ceux (bien nombreux) qui ne comprennent pas assez le français. Les décors et les costumes jouent le même rôle, d'où le budget important de ce théâtre. 
Les exemples de la faible aptitude des étrangers à comprendre le français «joué » sont légion. Markovits nous rappelle que Bruxelles est à l'époque une ville flamande où les francophones ne sont que $15 \%$ de la population. Quant à l'Italie, il cite une anecdote bien connue : à Bologne, en 1772, on ne s'aperçoit pas qu'au lieu de Zaïre de Voltaire, qui était initialement à l'affiche, on joue L'honnête criminel de Fenouillot de Falbaire. Et les Bolonais de s'extasier: "Oh, quelle tragédie! Oh, grand Voltaire!». Le décalage linguistique explique aussi la préférence donnée - sur les scènes hors de France - aux "pièces d'action", selon le témoignage de Ferdinando Galiani sur la tournée de comédiens français à Naples en 1773 ; dans une lettre à Madame d'Épinay, il établit une différence entre " pièce à dialogue » et " pièce à intrigue " : les Napolitains n'ont rien compris au Méchant de Grasset - explique-t-il à sa correspondante - parce que cette pièce « n'est que parlée » et que « rien ne s'y fait » (p. 104).

Un autre secours dans le cas d'une compréhension défaillante est le texte imprimé de la pièce que les spectateurs ont sur les genoux lors de la représentation, aide à la compréhension mais aussi vecteur efficace d'apprentissage de la langue française, comme le remarque encore Galiani qui nous peint les Napolitains les yeux baissés, " contents d'apprendre à lire le français » (ibid.). Ainsi le jeune Goethe qui, à l'âge de 12 ans, apprend très rapidement la langue de Paris grâce à la fréquentation assidue du théâtre français de Francfort, notamment des comédies - les pièces qui lui posent plus de difficultés : on y parle vite, remarque-t-il, et elles roulent sur les événements de la vie ordinaire que les étrangers connaissent mal (notons que la comédie est le genre le plus joué). Comme pour les Napolitains, pour Goethe, les textes imprimés interviennent. Par ailleurs, un certain nombre d'opérations s'imposent pour acclimater la culture française. Les pièces subissent de nombreux retranchements : réduction de la durée et du nombre des actes pour les exigences de la cour, et adaptations multiples. Durazzo suggère de supprimer les équivoques et, surtout, les traits scandaleux qui pourraient blesser la sensibilité des Viennois, un peuple aux mœurs simples : la satire contre le clergé, la galanterie, le bel esprit (p. 106). D’ailleurs, les particularismes liés à la situation politique ou sociale (l'argot, entre autres, dans les pièces du Théâtre de la foire) sont gommés. Le projet d'un théâtre aristocratique conforme au public de cour comporte un programme d'élimination du bas et l'élévation du registre de la langue. La sélection et l'adaptation qui se font à l'étranger fabriquent un nouveau théâtre. Le modèle de civilisation que s'approprie le spectateur hors de France est celui qui est offert par un spectacle aristocratique, épuré et moralisé. La difficulté des étrangers à comprendre le versant linguistique des pièces françaises n'est qu'un aspect d'un écart renvoyant aussi à une dimension culturelle non partagée. L'A. peut ainsi discuter plusieurs idées reçues sur l'Europe française : entre autres, précise-t-il, ce "mythe " met en avant les grandes figures francophones en faussant une réalité bien plus variable.

Un concept qui fait son chemin dans l'Europe du XVIII ${ }^{e}$ siècle est celui qu'on appellerait aujourd'hui impérialisme culturel : l'impérialisme culturel français - selon plusieurs, véhicule d'une francisation corruptrice - s'insinue à tous moments, notamment dans les rapports avec les pays les plus distants de la civilisation de l'Hexagone. Markovits consacre tout un chapitre à la querelle d'un théâtre dans Genève, la controverse sociolittéraire la plus connue, dans sa double formulation opposant Rousseau à D'Alembert et Voltaire au Consistoire genevois. Mais la perspective de l'A. est différente : elle met à jour "sa dimension proprement genevoise» (p. 198). Voltaire n'est pas le seul à assigner à la comédie le but de civiliser les «mœurs sauvages» des Genevois, mais 
Genève est le seul cas en Europe qui se ferme à la dissémination du théâtre français jusqu'en 1782 ; cas qui permet d'aligner identité française, civilisation et théâtre, dans une équivalence qui « finit par aboutir à l'idée d'une mission civilisatrice au nom de la supériorité culturelle française» (p. 245). L'hypothèse d'un impérialisme culturel se manifestant dans cette querelle mine encore une fois l'idée d'une diffusion spontanée de la culture française en Europe.

C'est encore d'un rapport supérieur/inférieur qu'il s'agit pour l'Italie aux yeux de Deleyre, encyclopédiste occupant à Parme les fonctions de bibliothécaire autour de 1765, qui regarde l'Italie comme un pays attardé. Markovits (p. 140-141) analyse le contexte et les réactions italiennes dans une guerre littéraire où du côté français se manifeste une forte agressivité, par exemple dans une lettre où Deleyre exprime son mépris à l'égard du théâtre italien, produit d'un pays décadent et arriéré. Mais, d'autre part, il peut nuancer la perspective de l'action civilisatrice au profit d'une autre perspective quand il se demande comment les pays étrangers se sont appropriés le répertoire et le modèle français véhiculé par le théâtre. Selon plusieurs observateurs italiens de l'époque, ce modèle peut contribuer à réformer les mœurs et les arts italiens : l'impérialisme culturel cède le pas à une hybridation culturelle des produits locaux. La tactique de mitigation dont se sert habituellement l'A. nuance ici la portée de l'Europe française en montrant que la propagation de la civilisation française n'est pas partout la même; elle se particularise, se localise en s'amalgamant aux cultures d'ailleurs. D'autres configurations se dessinent ainsi contre cette culture unique qu'aurait produite en Europe l'hégémonie culturelle de la France. Markovits peut également atténuer l'idée d'une francophilie européenne univoque, «à la fois dans le temps et dans l'espace » (p. 153).

11 L'impérialisme culturel que la France aspire à exercer à travers son théâtre se manifeste de manière très nette sous la Révolution et l'Empire. Instrument du pouvoir renvoyant à l'absolutisme louis-quatorzien, qui était, comme on le sait, un pouvoir à la fois politique et culturel, le théâtre - après avoir nourri, dans les mains de l'armée, l'aspiration à séduire par sa "puissance douce »- se pense comme une machine de guerre, quand on regarde l'autre comme un attardé qu'il faut guider sur la voie de la civilisation. Le cas de Bruxelles sous la Révolution est pris par Markovits comme emblème pour ce qui se passe un peu partout en Europe entre 1795 et 1814, quand le théâtre est mis au service de «l'acculturation des nouveaux Français» (p. 264) dans les départements annexés. Le chapitre 7 débute par la tournée de la troupe de la Montausier à Bruxelles où elle dit vouloir «propager les principes et l'amour de la liberté » parmi les Belges (lettre de 1793 au ministre des affaires étrangères). Cette fonction "pédagogique et prosélyte " (p. 248) du théatre auquel est confiée la tâche d'exporter les principes de la Révolution fait entrer en action la notion de propagande (c'est en effet de «troupe de propagande » que la Montausier qualifie ses comédiens). La même action peur être exercée par la langue, pensent les préfets napoléoniens aussi bien que les élites françaises, preuve en est la campagne mise en acte à Bruxelles contre le flamand, langue à extirper "par tous les moyens " (mémoire d'un fonctionnaire anonyme vers 1794). Cette politique linguistique agressive trouve une confirmation en Italie contre l'italien, "langue dégénérée des peuples du Latium» (Charron, commissaire général de police à Turin, dans un Rapport de 1802).

Plus de 60 pages de notes témoignent de la richesse de la bibliographie et des bases documentaires auxquelles Markovits a eu accès: d'abord les "classiques» sur la 
diffusion du français en Europe, puis des études générales sur la sociabilité et la conversation, la civilisation et la société de cour, la culture théâtrale et l'organisation des spectacles en Europe ; et encore des études locales sur des villes de l'Allemagne, de l'Italie, des Pays-Bas, de la Belgique. Ajoutons les archives et les nombreuses correspondances, les livres de mémoire - où les courtisans et les voyageurs enregistrent les spectacles auxquels ils assistent - et la presse périodique qui nous renseigne sur le répertoire français dans les différents pays. Grâce à ces sources l'A. a pu constituer une base de données de plus de 5000 titres de pièces.

Civilisation et culture, francisation et acculturation, hard power et soft power, propagation et dissémination, intégration et colonisation, impérialisme culturel et rhétorique de la libération, propagande et régénération, autant de notions que l'étude de Markovits sur les pérégrinations européennes du théâtre français au XVIII ${ }^{e}$ siècle nous invite à ré-explorer.

\section{AUTEUR}

NADIA MINERVA

Université de Catane, Italie 